\title{
Mu-Tau Neutrinos: Influencing Fast Flavor Conversions in Supernovae
}

\author{
Francesco Capozzi@ ${ }^{1,{ }^{*}}$ Madhurima Chakraborty, ${ }^{2, \uparrow}$ Sovan Chakraborty, ${ }^{2, \ddagger}$ and Manibrata Sen $\oplus^{3,4, \S}$ \\ ${ }^{1}$ Max-Planck-Institut für Physik (Werner-Heisenberg-Institut), Föhringer Ring 6, 80805 München, Germany \\ ${ }^{2}$ Indian Institute of Technology, Guwahati, Assam-781039, India \\ ${ }^{3}$ Department of Physics \& Astronomy, Northwestern University, 2145 Sheridan Road, Evanston, Illinois 60208, USA \\ ${ }^{4}$ Department of Physics, University of California Berkeley, Berkeley, California 94720, USA
}

(Received 20 June 2020; accepted 19 November 2020; published 16 December 2020)

\begin{abstract}
Neutrinos in a core-collapse supernova can undergo fast flavor conversions with a possible impact on the explosion mechanism and nucleosynthesis. We perform the first nonlinear simulations of fast conversions in the presence of three neutrino flavors. The recent supernova simulations with muon production call for such an analysis, as they relax the standard $\nu_{\mu, \tau}=\bar{\nu}_{\mu, \tau}$ (two-flavor) assumption. Our results show the significance of muon and tau lepton number angular distributions, together with the traditional electron lepton number ones. Indeed, our three-flavor results are potentially very different from two-flavor ones. These results strengthen the need to further investigate the occurrence of fast conversions in supernova simulation data, including the degeneracy breaking of mu and tau neutrinos.
\end{abstract}

DOI: 10.1103/PhysRevLett.125.251801

Introduction.-Neutrinos streaming out of a supernova (SN) encounter a large density of ambient neutrinos and antineutrinos. Forward scattering off this surrounding neutrino bath has been shown to cause self-induced effects, leading to collective flavor oscillations, where neutrinos with different oscillation frequencies change their flavors in a coherent fashion (see Refs. [1-4] for a comprehensive review). Recently, it was shown that under certain conditions, these collective oscillations can be "fast," growing with the extremely large neutrino density $n_{\nu}$, while being independent of the neutrino mass-squared difference $\Delta m^{2}$ or the neutrino energy $E$ [5-7]. These fast flavor conversions (FFC) can occur close to the region of neutrino free-streaming, and can grow as large as $10^{5}$ times faster than the vacuum oscillations.

A host of studies [5-20] in the past few years suggest that a necessary though not sufficient condition for the existence of these fast instabilities is the presence of a zero crossing in the angular distribution of the neutrino electron lepton number (ELN), i.e., the difference between the electron neutrino and the antineutrino angular emission spectra should go through a zero for some emission angle. Though neutrinos of other flavors are also present in the $\mathrm{SN}$ environment, ELN is considered to be the only driving quantity, as these studies are performed assuming an effective two-flavor scenario ( $e$ and $x$ flavor, where

Published by the American Physical Society under the terms of the Creative Commons Attribution 4.0 International license. Further distribution of this work must maintain attribution to the author(s) and the published article's title, journal citation, and DOI. Funded by SCOAP ${ }^{3}$. $x=\mu, \tau$ or a linear combination of both). This is due to the fact that in the absence of muons and taus in traditional SN simulations, the heavy lepton neutrinos have identical microphysics and similar number density $\left(n_{\nu_{\mu}}=n_{\nu_{\tau}}=\right.$ $n_{\bar{\nu}_{\mu}}=n_{\bar{\nu}_{\tau}}=n_{\nu_{x}}$ ) for all emission angles. Now, these crossings are naively expected to occur near the neutrino freestreaming zone and require a comprehensive study of the interplay of collisions and fast conversions, thereby necessitating the use of quantum kinetic equations [21-24]. Recently, dedicated studies have performed a thorough scan of existing data from SN hydrodynamical simulations. In Ref. [25] it was shown that crossings in the ELN, sometimes related to the lepton number emission selfsustained asymmetry (LESA) [26,27], are present both in the convective layer of the proto-neutron star (PNS) and outside the neutrino decoupling region. Such results have been confirmed with further insights from Ref. [18]. Furthermore, recently it was found [28] that coherent neutrino-nucleus scattering in the pre-shock region can lead to a tiny ELN zero crossing in the backward direction, leading to FFC at a distance of $\mathcal{O}(100) \mathrm{km}$ from the PNS.

The presence of these recently discovered FFCs well inside a $\mathrm{SN}$ can lead to paradigm changes in our understanding of the explosion dynamics, requiring a more detailed analysis of the different approximations going into the studies. In particular, the above results are based on the effective two-flavor setup. This setup has severe limitations as the $\nu_{x}$ and $\bar{\nu}_{x}$ flux would differ naturally if nucleon recoil effects, implying different neutral-current scattering cross sections, are taken into account. Moreover, it would be also natural to expect that the high temperatures during the accretion phase would create muons in the 
nascent neutron stars. In fact, Ref. [29] has shown that the addition of muons can enhance neutrino energy deposition to the stalled shockwave, leading to a successful explosion. Muon production in a SN [29] can create differences in the heavy lepton flavor neutrino fluxes, thereby necessitating the inclusion of three-flavor effects. The oscillation treatment of such a scenario was pointed out in Ref. [30] and recently, Ref. [31] has done a detailed analysis of fast conversions to three neutrino flavors. Using a linear stability analysis, it demonstrated the possibility of altering the instability growth rates obtained in the standard twoflavor setup.

Going a step forward with respect to current literature, we, for the first time, perform a fully nonlinear computation of FFCs in the presence of three neutrino flavors. Motivated by the difference in the heavy lepton flavor neutrino fluxes observed in Ref. [29], we propose simple toy models and demonstrate that it is not only the ELN, but also $\mu \mathrm{LN}$ and $\tau \mathrm{LN}$ that drives the onset of these rapid conversions. In fact, it is the difference of any of the two-flavor lepton numbers that goes into the evolution equations [31]. This emphasizes the incompleteness of a two-flavor analysis, especially while analyzing the presence of FFC in the preshock region, where tiny crossings in the ELN can get erased by the $\mu \mathrm{LN}$ and or $\tau \mathrm{LN}$. Our results suggest the importance of such a fully nonlinear three-flavor study of FFC with detailed hydrodynamic SN simulations, including muon production, to gauge its impact on SN dynamics.

Equations of motion.-The dynamics of the neutrino mean field, described by matrices of neutrino density $\rho_{\mathbf{p}, \mathbf{x}, t}$ for momentum $\mathbf{p}$ at position $\mathbf{x}$ and time $t$, is governed by the equations of motion (EOMs) [32]

$$
i\left(\partial_{t}+\mathbf{v}_{\mathbf{p}} \cdot \nabla_{\mathbf{x}}\right) \rho_{\mathbf{p}, \mathbf{x}, t}=\left[\Omega_{\mathbf{p}, \mathbf{x}, t}, \rho_{\mathbf{p}, \mathbf{x}, t}\right],
$$

where the left-hand side accounts for time and spatial dependence of $\rho_{\mathbf{p}, \mathbf{x}, t}$. The diagonal elements of $\rho_{\mathbf{p}, \mathbf{x}, t}$ give the survival probability of a given flavor, whereas the offdiagonal elements encode flavor coherence. The Hamiltonian $\Omega_{\mathbf{p}, \mathbf{x}, t}$ on the right-hand side consists of the vacuum term $\left(\Delta m^{2} / 2 E\right)$, the Mikheyev-Smirnov-Wolfenstein matter potential, $\lambda_{\alpha}=\sqrt{2} G_{F} n_{\alpha}$, depending on the background charged lepton number density $n_{\alpha}$, and the $\nu-\nu$ selfinteraction potential, $\mu \int d^{3} \mathbf{q} /(2 \pi)^{3}\left(1-\mathbf{v}_{\mathbf{p}} \cdot \mathbf{v}_{\mathbf{q}}\right)\left(\rho_{\mathbf{q}, \mathbf{x}, t}-\bar{\rho}_{\mathbf{q}, \mathbf{x}, t}\right)$, where $\mu=\sqrt{2} G_{F} n_{\nu}$ for a background neutrino density $n_{\nu}$ [2]. Here $G_{F}$ refers to the Fermi constant.

In the context of fast oscillations, the vacuum term does not play a major role, except for seeding the oscillations. For a two-flavor FFC computation with both spatial and temporal evolution, one can neglect the matter term due to background electron density since it generally does not affect the growth rate of oscillations, and only delays the onset logarithmically [14] (see Ref. [33] for a detailed analysis on the role of background matter), whereas in a three-flavor setup with both electrons and muons in the background, this is not trivial [34,35]. However, our numerical examples only deal with time evolution and the matter terms can be safely neglected [7]. Thus, only the nonlinear term governs the dynamics of our setup. Note that, due to the presence of three flavors there can be radiative corrections in this term. However, these are small and can be dropped [35]. What enters the EOMs is the difference in the occupation numbers of the different flavors integrated over energy, dubbed in literature as the corresponding neutrino flavor lepton number. In the twoflavor scenario, assuming the nonelectron flavor angular distributions are identical for both the particles and the antiparticles, the electron lepton number (ELN) is defined as the difference between the $\nu_{e}$ and the $\bar{\nu}_{e}$ distribution, integrated over energy, [10]

$$
G_{\mathbf{v}}^{e}=\sqrt{2} G_{F} \int_{0}^{\infty} \frac{d E E^{2}}{2 \pi^{2}}\left[\rho_{e e}(E, \mathbf{v})-\bar{\rho}_{e e}(E, \mathbf{v})\right] .
$$

However, 2D simulations [29] show that this picture is not entirely accurate; the inclusion of muons does create an appreciable flux hierarchy between the $\nu_{\mu}$ and $\bar{\nu}_{\mu}$ in the accretion phase. This is primarily because muons can be pair produced from electrons, and can participate in $\beta$ processes to create $\nu_{\mu}$ and $\bar{\nu}_{\mu}$. The relative neutron-toproton ratio governs the extent of muonization $\left(\nu_{\mu}-\bar{\nu}_{\mu}\right)$ in the PNS. In contrast, due to the high value of $m_{\tau}$, the $\tau$ density remains small throughout, although a tiny asymmetry may arise due to different scattering cross sections with nucleons.

Thus, within the three-flavor formalism [30,31], one needs to define the corresponding muon lepton number $(\mathrm{MuLN})$ and tau lepton number (TauLN) as

$$
\begin{aligned}
G_{\mathbf{v}}^{\mu} & =\sqrt{2} G_{F} \int_{0}^{\infty} \frac{d E E^{2}}{2 \pi^{2}}\left[\rho_{\mu \mu}(E, \mathbf{v})-\bar{\rho}_{\mu \mu}(E, \mathbf{v})\right], \\
G_{\mathbf{v}}^{\tau} & =\sqrt{2} G_{F} \int_{0}^{\infty} \frac{d E E^{2}}{2 \pi^{2}}\left[\rho_{\tau \tau}(E, \mathbf{v})-\bar{\rho}_{\tau \tau}(E, \mathbf{v})\right] .
\end{aligned}
$$

The EOM is sensitive to the difference of these lepton numbers via the following quantities,

$$
\begin{aligned}
& G_{\mathbf{v}}^{e \mu}=G_{\mathbf{v}}^{e}-G_{\mathbf{v}}^{\mu}, \\
& G_{\mathbf{v}}^{e \tau}=G_{\mathbf{v}}^{e}-G_{\mathbf{v}}^{\tau}, \\
& G_{\mathbf{v}}^{\mu \tau}=G_{\mathbf{v}}^{\mu}-G_{\mathbf{v}}^{\tau} .
\end{aligned}
$$

Note that $G_{\mathbf{v}}^{e \mu}$ reduces to the ELN in the two-flavor scenario, where $n_{\nu_{\mu}}=n_{\bar{\nu}_{\mu}}$. There exists three off-diagonal elements of the density matrix: $\rho_{e \mu}, \rho_{e \tau}, \rho_{\mu \tau}$, which might undergo an exponential growth (i.e., an instability), when a crossing is created in one of the angular distributions. Therefore, a crossing in the ELN is not enough, since one 
needs to consider $G^{e \mu}, G^{e \tau}$, or $G^{\mu \tau}$ [31]. This has important consequences for FFC. For instance, a tiny crossing in the ELN can be erased by a negative MuLN. Analogously, the absence of a crossing in the ELN can be compensated by a positive MuLN. Similar arguments hold for the other sectors. As a result, claiming the presence of fast oscillations, focusing only on tiny ELN crossings, as done for example in Ref. [28], might lead to incomplete conclusions. In what follows, we consider simple toy models to demonstrate this important point.

Numerical examples.-We assume a spatially homogeneous flavor composition, and only consider the time evolution in Eq. (1). We assume that the initial neutrino angular distributions are axially symmetric around the $z$ axis, i.e., they only depend on the zenith angle. Nevertheless, we do consider the azimuthal angles in our calculations, so that also axially breaking instabilities are allowed to develop [36]. We take $\mu=4 \times 10^{5} \mathrm{~km}^{-1}$, which is a typical value in the neutrino decoupling region. Concerning the vacuum term, we use as oscillation frequencies $\Delta m_{31}^{2} /(2 E)=0.5$ and $\Delta m_{21}^{2} /(2 E)=0.01 \mathrm{~km}^{-1}$, whereas we set the mixing angles to be $\theta_{12}=\theta_{13}=\theta_{23}=10^{-3}$. In this way, we mimic the suppression of $\theta_{i j}$ induced by the large potentials. Moreover, as we focus only on the time evolution we neglect the matter terms, $\lambda_{e}=\lambda_{\mu}=\lambda_{\tau}=0$.

In the following, we consider four toy cases of $G_{\mathbf{v}}^{e \mu}, G_{\mathbf{v}}^{e \tau}$, and $G_{\mathbf{v}}^{\mu \tau}$, highlighting the fact that the effective two-flavor formalism leads to different conclusions. Note that current state of the art SN simulations [29] can only provide angular moments of the neutrino distribution, from which one can construct realistic angle dependence of the neutrino flavor intensity but only near the neutrinosphere. However, these distributions are not very reliable at larger radii [37]. We leave to future work the assessment of which lepton number angular distributions are realized in nature.

First, we consider the scenario in Fig. 1, where each of $G_{\mathbf{v}}^{e}, G_{\mathbf{v}}^{\tau}$, and $G_{\mathbf{v}}^{\mu}$ have crossings as can be interpreted from the angular distribution of the fluxes (uppermost panel). We show the $G_{\mathbf{v}}^{e}$ crossing by the dashed lines in the ' $G_{\mathbf{v}}^{\alpha \beta}$ vs $\mathbf{v}$ ' panel. Thus, in the two-flavor scenario $\left(n_{\nu_{\mu}}=n_{\bar{\nu}_{\mu}}\right)$, there is an exponential growth in $\rho_{e \mu}$ (dashed line, lower panel), due to this crossing in $G_{\mathbf{v}}^{e}$. However, it is evident that no crossing persists in $G_{\mathbf{v}}^{e \mu}, G_{\mathbf{v}}^{e \tau}$, or $G_{\mathbf{v}}^{\mu \tau}$. Consequently, the time evolution of the off-diagonal elements of $\left|\left\langle\rho_{\alpha \beta}\right\rangle\right|$ (solid lines, lower panel) does not show any exponential growth. Here, we have defined

$$
\left|\left\langle\rho_{\alpha \beta}\right\rangle\right|=\left|\int d \mathbf{v} \rho_{\alpha \beta}(\mathbf{v})\right|
$$

This simple but crucial example clearly demonstrates that some of the crossings found in Refs. [18,25,28] might disappear once the corresponding hydrodynamical
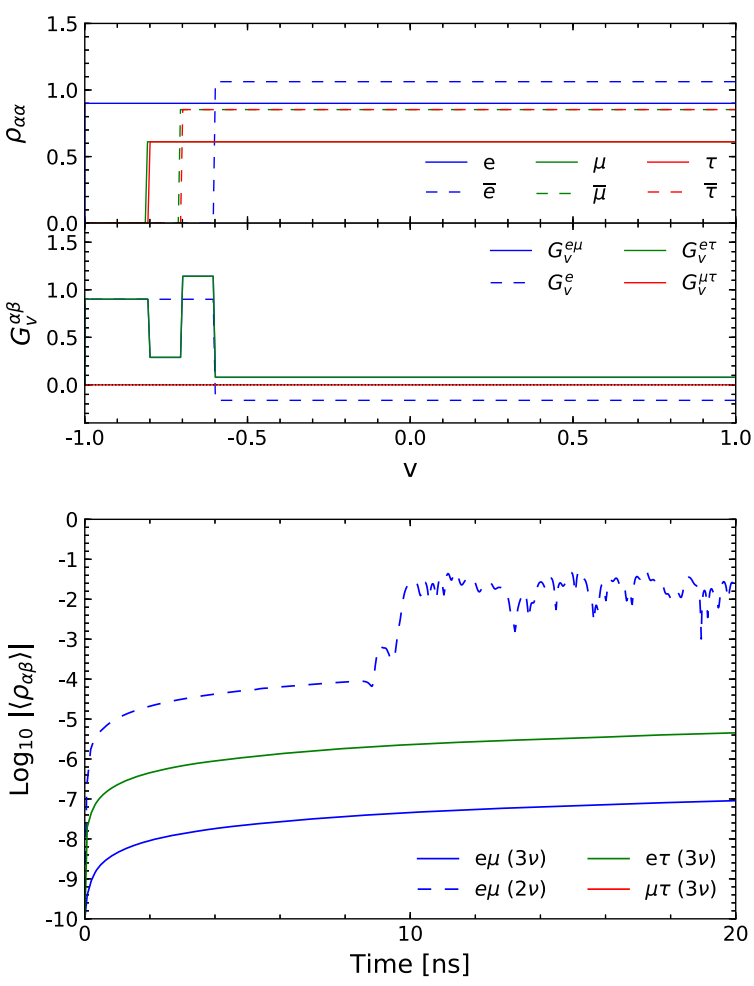

FIG. 1. The upper panels show the angular distribution of the fluxes and the effective lepton numbers for different flavors. The effective lepton number panel $\left(G_{\mathbf{v}}^{\alpha \beta}\right.$ vs $\left.\mathbf{v}\right)$ shows both the three-flavor (solid lines) and the two-flavor ELN (dashed line). The lower panel shows the evolution of the angle-averaged offdiagonal elements $\left|\left\langle\rho_{\alpha \beta}\right\rangle\right|$ of the density matrix with time (in ns). The three-flavor evolution (solid lines) has no instability, while the effective two-flavor evolution, assuming $\nu_{x}=\bar{\nu}_{x}$, shows large exponential growth (dashed line).

simulation include the full three-flavor neutrino transport, including the production of muons.

As a second example, consider Fig. 2 (upper panels), where the $\operatorname{ELN}\left(G_{\mathbf{v}}^{e}\right)$ has a regular crossing (dashed line), but there are none in the MuLN or the TauLN. The spectra are designed such that the flavor lepton number difference $G_{\mathbf{v}}^{e \mu}, G_{\mathbf{v}}^{e \tau}$ exhibit deep crossings (solid lines). On the other hand, the crossing in $G_{\mathbf{v}}^{\mu \tau}$ is extremely shallow. The naive two-flavor intuition is that there should be exponential growths in the $e-\mu$ and $e-\tau$ sector. However, the nonlinear, coupled nature of the problem intertwines the growths in all the sectors. This is borne by the lower panel, where substantial flavor conversion is seen in all the sectors, though with different growth rates. Note that the growth in $\rho_{\mu \tau}$ is inherently a nonlinear effect, and will not be captured by a linear stability analysis. The corresponding two-flavor evolution of $\rho_{e \mu}$ is also shown for comparison. Clearly, the two-flavor evolution is very different, with a larger onset time and growth rate.

The third case, shown in Fig. 3, presents a crossing only in $G_{\mathbf{v}}^{\mu \tau}$. There exists a reasonable asymmetry (upper panels) 

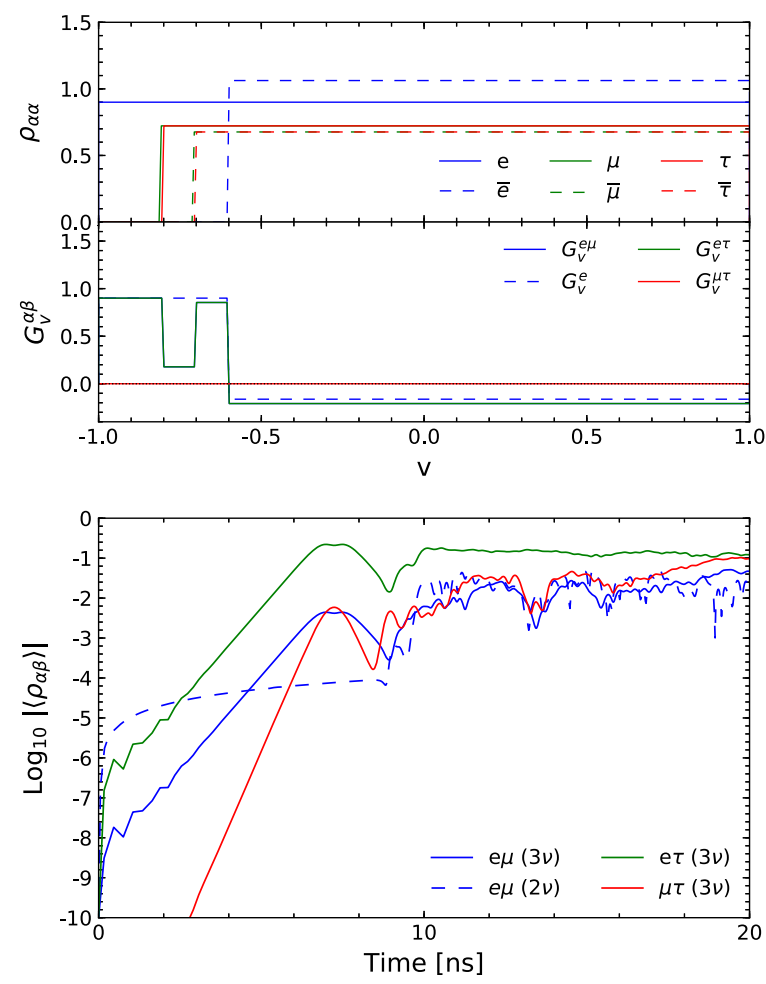

FIG. 2. Panels represent same as in Fig. 1. However, due to large crossings in $G_{\mathbf{v}}^{e \mu}, G_{\mathbf{v}}^{e \tau}$, substantial flavor conversions are seen in all the sectors.
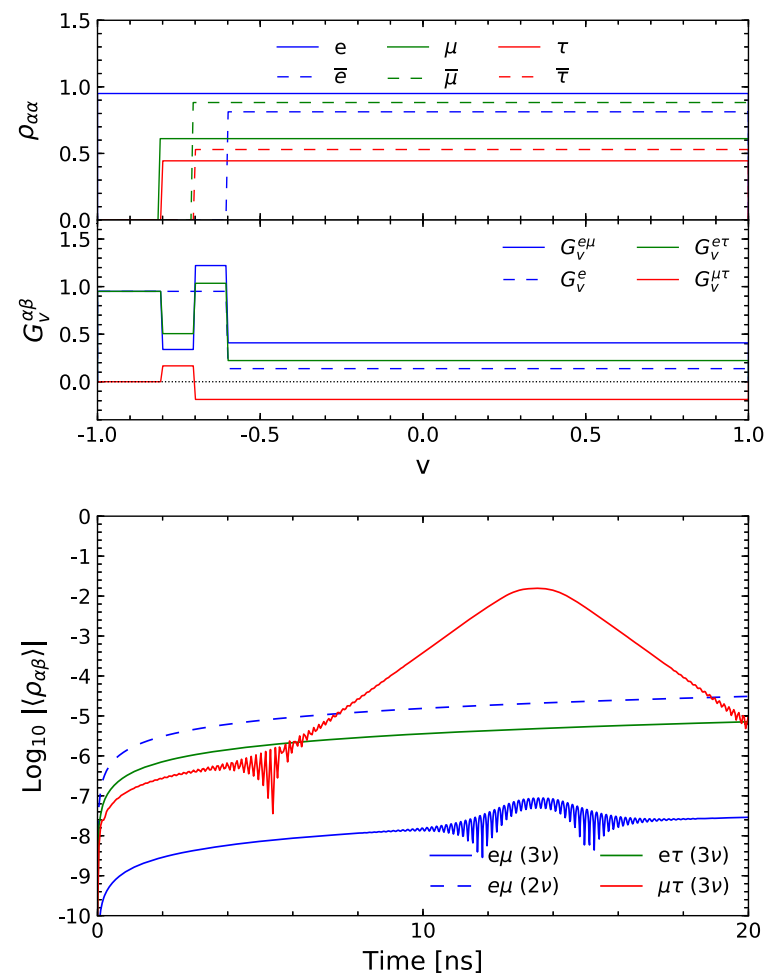

FIG. 3. Panels represent same as in Fig. 1. Crossing only in $G_{\mathbf{v}}^{\mu \tau}$ causes an exponential growth in $\mu-\tau$ sector (lower panel). The two-flavor evolution shows no instability. between $\nu_{\mu}$ and $\nu_{\tau}$ (and between their antiparticles as well) to generate an exponential growth in the $\mu-\tau$ sector. This is what is seen in the lower panel, where the $\mu-\tau$ sector experiences a flavor instability, while the other two do not. Indeed, the two-flavor analysis (dashed line) also does not exhibit any instability due to the lack of a crossing in the $\operatorname{ELN}\left(G_{\mathbf{v}}^{e}\right)$. This example advances the hypothesis that those regions where no ELN crossing was found in $[18,25,28]$ might, in reality, have fast instabilities once the differences between $\nu_{\mu}$ and $\bar{\nu}_{\mu}$ are taken into account. Another comment is in order: the amplitude of the exponential growth in our toy model is not enough to cause substantial flavor conversions. However, the background conditions for these solutions may dynamically change in a realistic SN environment, or if spatial evolution is taken into account, and may result in flavor conversions.

As a final example, we consider a scenario in Fig. 4 where shallow crossings are present in $G_{\mathbf{v}}^{e \mu}, G_{\mathbf{v}}^{e \tau}$ in the backward direction, whereas $G_{\mathbf{v}}^{\mu \tau}$ shows a significant crossing in the forward direction as well. To contrast with the two-flavor examples, the setup is constructed such that the $\operatorname{ELN}\left(G_{\mathbf{v}}^{e}\right)$ also has a shallow crossing but in the forward direction only. We find that such shallow crossings readily lead to an instability in the three-flavor case, whereas the two-flavor setup shows no instability (lower panel).
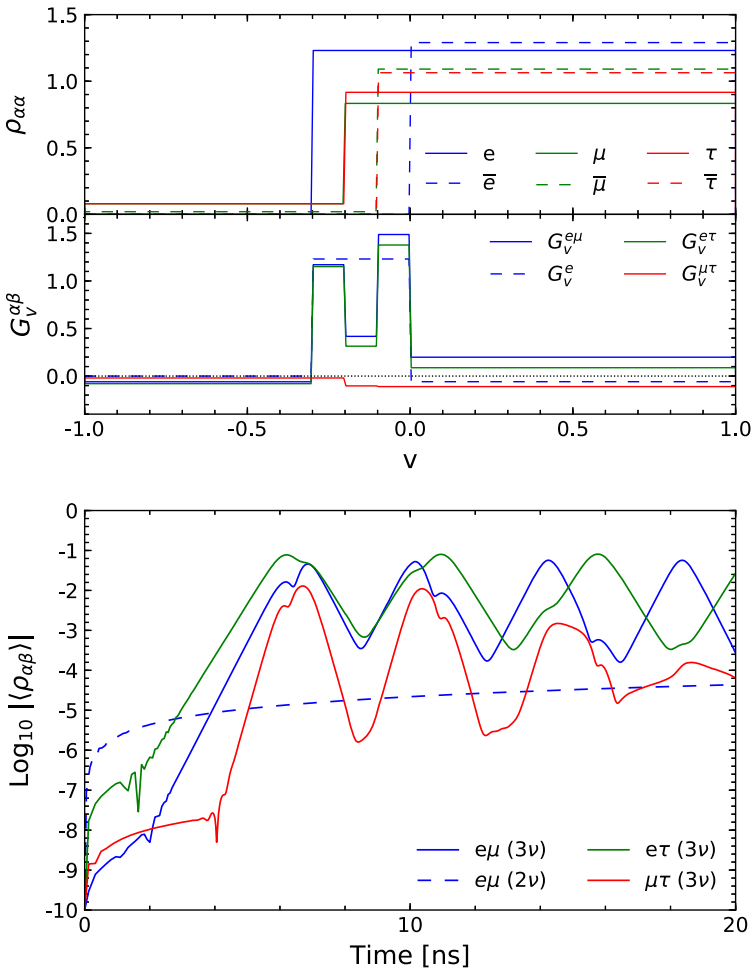

FIG. 4. Panels represent same as in Fig. 1. However, shallow crossings are present in $G_{\mathbf{v}}^{e \mu}, G_{\mathbf{v}}^{e \tau}$ in the backward direction (upper panels), leading to exponential growths in $\left|\left\langle\rho_{\alpha \beta}\right\rangle\right|$ (lower panel) for the three-flavor setup. The two-flavor evolution shows no instability. 
This example is motivated from Ref. [28], where it was pointed out that shallow crossings in the backward directions can lead to a fast instability. In Ref. [28], such backward crossings were associated with residual coherent scattering on heavy nuclei, which is slightly enhanced for $\bar{\nu}_{e}$ with respect to $\nu_{e}$, because of their larger average energy. Our toy model advances the hypothesis that the existing differences between $\nu_{\mu}$ and $\bar{\nu}_{\mu}$ could (at least in principle) be the real cause of these crossings. Similarly, the nonnegligible muon lepton number can also erase a potential shallow (backward) crossing in the ELN. Our examples clearly establish the importance of detailed three-flavor treatments to assess whether such possibilities are indeed realized in nature. Future muon SN simulations will hold the key to the answer.

Discussion and conclusions.-Fast neutrino flavor conversion near the SN core can lead to a paradigm change in our understanding of flavor evolution of supernova neutrinos. Within a two-flavor formalism, these ultrarapid flavor conversions are believed to occur mainly when the ELN, i.e., the difference between the $\nu_{e}$ and $\bar{\nu}_{e}$ angular spectra, exhibit a zero crossing. Such flavor mixing can have a drastic impact on the shockwave revival, as well as nucleosynthesis. Hence, it is crucial to appreciate these flavor conversions, using a complete three-flavor analysis.

We have performed, for the first time, a completely nonlinear, three-flavor treatment of fast flavor conversions of neutrinos. We find that the inclusion of three flavors can significantly alter our understanding of the conditions for fast conversions. Using simple toy spectra, we demonstrate that it is not the ELN, or correspondingly, the MuLN, and TauLN, but rather their differences that govern these fast modes. The examples studied in this paper clearly show that three-flavor evolution can result in instabilities that are not captured by a two-flavor study; conversely, it can also wash out the instabilities predicted by a two-flavor study. These results also show that the linear stability analysis cannot capture all the instability signatures of the full nonlinear analysis.

Our findings further indicate caution against claiming the presence of fast conversions for shallow crossings in the ELN, because such crossings can easily be nullified by an opposite crossing in the MuLN. This is particularly relevant in the shock region, where a significant population of $\nu_{\mu}, \nu_{\tau}$ can be expected in the accretion phase. This motivates the necessity of including muons in a dedicated analysis of fastflavor conversions to gauge their impact on supernova dynamics.

We would like to thank Georg Raffelt, Alessandro Mirizzi, Basudeb Dasgupta, and Sajad Abbar for useful comments on the manuscript. M.S. acknowledges support from the National Science Foundation, Grant No. PHY-1630782, and to the Heising-Simons Foundation, Grant No. 2017228. The work of F. C. is supported by the Deutsche Forschungsgemeinschaft through Grants SFB-1258
"Neutrinos and Dark Matter in Astro- and Particle Physics (NDM)" and EXC 2094 "ORIGINS: From the Origin of the Universe to the First Building Blocks of Life." S. C. acknowledges the support of the Max Planck India Mobility Grant from the Max Planck Society. M. C. and S. C. also received support from the European Union's Horizon 2020 research and innovation programme through the InvisiblesPlus RISE and Elusives ITN under the Marie Skłodowska-Curie Grant Agreement No. 690575 and Agreement No. 674896, respectively

*capozzi@mppmu.mpg.de

†madhu176121012@iitg.ac.in

†sovan@iitg.ac.in

§manibrata@berkeley.edu

[1] H. Duan, G. M. Fuller, and Y.-Z. Qian, Collective neutrino oscillations, Annu. Rev. Nucl. Part. Sci. 60, 569 (2010).

[2] A. Mirizzi, I. Tamborra, H.-T. Janka, N. Saviano, K. Scholberg, R. Bollig, L. Hüdepohl, and S. Chakraborty, Supernova neutrinos: Production, oscillations and detection, Riv. Nuovo Cimento 39, 1 (2016).

[3] S. Chakraborty, R. Hansen, I. Izaguirre, and G. G. Raffelt, Collective neutrino flavor conversion: Recent developments, Nucl. Phys. B908, 366 (2016).

[4] S. Horiuchi and J. P. Kneller, What can be learned from a future supernova neutrino detection? J. Phys. G 45, 043002 (2018).

[5] R. F. Sawyer, Neutrino Cloud Instabilities just above the Neutrino Sphere of a Supernova, Phys. Rev. Lett. 116, 081101 (2016).

[6] S. Chakraborty, R. S. Hansen, I. Izaguirre, and G. G. Raffelt, Self-induced neutrino flavor conversion without flavor mixing, J. Cosmol. Astropart. Phys. 03 (2016) 042.

[7] B. Dasgupta, A. Mirizzi, and M. Sen, Fast neutrino flavor conversions near the supernova core with realistic flavordependent angular distributions, J. Cosmol. Astropart. Phys. 02 (2017) 019. [

[8] R. F. Sawyer, Speed-up of neutrino transformations in a supernova environment, Phys. Rev. D 72, 045003 (2005).

[9] R. F. Sawyer, The multi-angle instability in dense neutrino systems, Phys. Rev. D 79, 105003 (2009).

[10] I. Izaguirre, G. G. Raffelt, and I. Tamborra, Fast Pairwise Conversion of Supernova Neutrinos: A Dispersion-Relation Approach, Phys. Rev. Lett. 118, 021101 (2017).

[11] F. Capozzi, B. Dasgupta, E. Lisi, A. Marrone, and A. Mirizzi, Fast flavor conversions of supernova neutrinos: Classifying instabilities via dispersion relations, Phys. Rev. D 96, 043016 (2017).

[12] A. Dighe and M. Sen, Nonstandard neutrino self-interactions in a supernova and fast flavor conversions, Phys. Rev. D 97, 043011 (2018).

[13] B. Dasgupta and M. Sen, Fast neutrino flavor conversion as oscillations in a quartic potential, Phys. Rev. D 97, 023017 (2018).

[14] B. Dasgupta, A. Mirizzi, and M. Sen, Simple method of diagnosing fast flavor conversions of supernova neutrinos, Phys. Rev. D 98, 103001 (2018). 
[15] S. Abbar, H. Duan, K. Sumiyoshi, T. Takiwaki, and M. C. Volpe, On the occurrence of fast neutrino flavor conversions in multidimensional supernova models, Phys. Rev. D 100, 043004 (2019).

[16] M. D. Azari, S. Yamada, T. Morinaga, W. Iwakami, H. Nagakura, and K. Sumiyoshi, Linear analysis of fastpairwise collective neutrino oscillations in core-collapse supernovae based on the results of Boltzmann simulations, Phys. Rev. D 99, 103011 (2019).

[17] L. Johns, H. Nagakura, G. M. Fuller, and A. Burrows, Neutrino oscillations in supernovae: Angular moments and fast instabilities, Phys. Rev. D 101, 043009 (2020).

[18] R. Glas, H. T. Janka, F. Capozzi, M. Sen, B. Dasgupta, A. Mirizzi, and G. Sigl, Fast neutrino flavor instability in the neutron-star convection layer of three-dimensional supernova models, Phys. Rev. D 101, 063001 (2020).

[19] S. Shalgar, I. Padilla-Gay, and I. Tamborra, Neutrino propagation hinders fast pairwise flavor conversions, J. Cosmol. Astropart. Phys. 06 (2020) 048.

[20] S. Bhattacharyya and B. Dasgupta, Fast neutrino flavor conversion at late time, Phys. Rev. D 102, 063018 (2020).

[21] A. Vlasenko, G. M. Fuller, and V. Cirigliano, Neutrino quantum kinetics, Phys. Rev. D 89, 105004 (2014).

[22] C. Volpe, Neutrino quantum kinetic equations, Int. J. Mod. Phys. E 24, 1541009 (2015).

[23] F. Capozzi, B. Dasgupta, A. Mirizzi, M. Sen, and G. Sigl, Collisional Triggering of Fast Flavor Conversions of Supernova Neutrinos, Phys. Rev. Lett. 122, 091101 (2019).

[24] S. A. Richers, G. C. McLaughlin, J. P. Kneller, and A. Vlasenko, Neutrino quantum kinetics in compact objects, Phys. Rev. D 99, 123014 (2019).

[25] S. Abbar, H. Duan, K. Sumiyoshi, T. Takiwaki, and M. C. Volpe, Fast neutrino flavor conversion modes in multidimensional core-collapse supernova models: The role of the asymmetric neutrino distributions, Phys. Rev. D 101, 043016 (2020).

[26] I. Tamborra, F. Hanke, H.-T. Janka, B. Müller, G. G. Raffelt, and A. Marek, Self-sustained asymmetry of lepton-number emission: A new phenomenon during the supernova shockaccretion phase in three dimensions, Astrophys. J. 792, 96 (2014).

[27] R. Glas, H. T. Janka, T. Melson, G. Stockinger, and O. Just, Effects of LESA in three-dimensional supernova simulations with multi-dimensional and ray-by-ray-plus neutrino transport, Astrophys. J. 881, 36 (2019).

[28] T. Morinaga, H. Nagakura, C. Kato, and S. Yamada, Fast neutrino-flavor conversion in the preshock region of corecollapse supernovae, Phys. Rev. Research 2, 012046 (2020).

[29] R. Bollig, H. T. Janka, A. Lohs, G. Martinez-Pinedo, C. J. Horowitz, and T. Melson, Muon Creation in Supernova Matter Facilitates Neutrino-Driven Explosions, Phys. Rev. Lett. 119, 242702 (2017).

[30] S. Airen, F. Capozzi, S. Chakraborty, B. Dasgupta, G. Raffelt, and T. Stirner, Normal-mode analysis for collective neutrino oscillations, J. Cosmol. Astropart. Phys. 12 (2018) 019.

[31] M. Chakraborty and S. Chakraborty, Three flavor neutrino conversions in supernovae: Slow \& fast instabilities, J. Cosmol. Astropart. Phys. 01 (2020) 005.

[32] G. Sigl and G. G. Raffelt, General kinetic description of relativistic mixed neutrinos, Nucl. Phys. B406, 423 (1993).

[33] S. Abbar and H. Duan, Fast neutrino flavor conversion: Roles of dense matter and spectrum crossing, Phys. Rev. D 98, 043014 (2018).

[34] A. Esteban-Pretel, S. Pastor, R. Tomas, G. G. Raffelt, and G. Sigl, Mu-tau neutrino refraction and collective three-flavor transformations in supernovae, Phys. Rev. D 77, 065024 (2008).

[35] A. Mirizzi, S. Pozzorini, G. G. Raffelt, and P. D. Serpico, Flavour-dependent radiative correction to neutrino-neutrino refraction, J. High Energy Phys. 10 (2009) 020.

[36] G. Raffelt, S. Sarikas, and D. de Sousa Seixas, Axial Symmetry Breaking in Self-Induced Flavor Conversion of Supernova Neutrino Fluxes, Phys. Rev. Lett. 111, 091101 (2013); Erratum, Phys. Rev. Lett. 113, 239903 (2014).

[37] T. Janka and R. Bollig (private communication). 